\title{
Phase transition dynamics in hot nuclei and N/Z influence
}

\author{
Bernard Borderie ${ }^{1, *}$, Nicolas Le Neindre ${ }^{2}$, and Pierre Désesquelles ${ }^{3}$ for the INDRA Collaboration \\ ${ }^{1}$ Institut de Physique Nucléaire, CNRS/IN2P3, Univ. Paris-Sud, Université Paris-Saclay, Orsay, France \\ ${ }^{2}$ Normandie Univ., ENSICAEN, UNICAEN, CNRS/IN2P3, LPC Caen, Caen, France \\ ${ }^{3}$ CSNSM, CNRS/IN2P3, Univ. Paris-Sud, Université Paris-Saclay, Orsay, France
}

\begin{abstract}
An abnormal production of events with almost equal-sized fragments was theoretically proposed as a signature of spinodal instabilities responsible for nuclear multifragmentation in the Fermi energy domain. On the other hand finite size effects are predicted to strongly reduce this extra production. High statistics quasifusion hot nuclei produced in central collisions between Xe and $\mathrm{Sn}$ isotopes at 32 and $45 \mathrm{MeV}$ per nucleon incident energies have been used to definitively establish, through the experimental measurement of charge correlations, the presence of spinodal instabilities. N/Z influence was also studied. The nature of the phase transition dynamics i.e. the fragment formation was the last missing piece of the puzzle concerning the liquidgas transition in nuclei.
\end{abstract}

\section{Introduction}

An important challenge of heavy-ion collisions at intermediate energies was the identification and characterization of the nuclear liquid-gas phase transition in hot nuclei, which has been theoretically predicted for nuclear matter. At present one can say that huge progress has been made even if some points can be deeper investigated $[1,2]$. Statistical mechanics for finite systems appeared as a key issue to progress, revealing specific first-order phase transition signatures related to the consequences of the local convexity of the entropy (see [2] and references therein). By considering the microcanonical ensemble with energy as extensive variable, the convex intruder implies a backbending in the temperature (first derivative of entropy) at constant pressure [3] and correlatively a negative branch for the heat capacity (second derivative of entropy). Experimentally, these two converging signatures have been observed in hot nuclei from different analyses [4-7] of homogeneous event samples. It is important to recall here that signals of phase transition for finite systems are only meaningful at the level of statistical ensembles constructed from the outcome of carefully selected collisions. Another consequence of the entropy curvature anomaly manifests itself when systems are treated in the canonical ensemble. In this case a direct phase transition signature is the presence of a bimodal distribution of an order parameter [8] such as the charge (size) of the largest fragment $\left(\mathrm{Z}_{\max }\right)$ of multifragmentation partitions [9].

The knowledge of the nature of the dynamics involved during phase transition in hot nuclei, i.e. fragment formation, is certainly the most delicate point. Two mechanisms have been proposed. On one side, stochastic mean field approaches predict the transition dynamics to follow the

*e-mail: borderie@ipno.in2p3.fr spinodal fragmentation scenario proposed very early on, triggered by phase-space fluctuations amplified in an unstable medium $[10,11]$ and, on the other side, molecular dynamics models such as AMD in which many-body correlations are sufficient to produce fragments at early times even in absence of unstable conditions [12, 13]. Both theoretical and experimental studies have evidenced for a radial extra energy boost (radial expansion energy) associated to multifragmentation products $[14,15]$. It can be attributed either to a dominant compression-expansion phase in central nucleus-nucleus collisions or to thermal pressure for more gentle collisions: hadron-nucleus or semi-peripheral nucleus-nucleus collisions. The system might then reach densities and temperatures that correspond to the unstable spinodal region where exponential amplification of density fluctuations leads to clusterization: an inhomogeneous mixture of fragments (normal density region), nucleons and light fragments (low density region). This can be seen as an analogue for phase separation in a finite system, for which spinodal decomposition would be the microscopic mechanism.

\section{Theoretical predictions}

In infinite nuclear matter the signature of spinodal instabilities is the formation of equal-sized fragments due to density fluctuations which grow exponentially with time. The most unstable modes correspond to wavelengths lying around $\lambda \approx 10 \mathrm{fm}$ and the associated characteristic times are equal to around $30-50 \mathrm{fm} / \mathrm{c}$, depending on density $\left(\rho_{0} / 2-\rho_{0} / 8\right)$ and temperature $(0-9 \mathrm{MeV})[10,11]$. A direct consequence of the dispersion relation is the production of "primitive" fragments with size $\lambda / 2 \approx 5 \mathrm{fm}$ which correspond to $Z \approx 8-10$. However, this simple picture is expected to be largely blurred by several effects. The 
beating of different modes occurs. Coalescence effects due to the nuclear interaction between fragments before the complete disassembly are also expected. For finite systems the situation is even more complicated. The presence of a surface introduces an explicit breaking of the translational symmetry with the important result that the growth rates are nearly the same for different multipolarities, which indicates that the unstable finite system breaks into different channels depending on multipolarity $L$ [16]. Equal-sized "primitive" fragments are then expected to be produced with sizes in the range $A_{F} / 2-A_{F} / L_{\text {max }} ; A_{F}$ being the part of the system leading to fragments during the spinodal fragmentation. Moreover the finite system produced during the nucleus-nucleus collision has to stay or live long enough in the spinodal region $(\sim 3$ characteristic time - $100-150 \mathrm{fm} / \mathrm{c}$ - for rather symmetric hot nuclei and - 200-300 fm/c - for nuclei with large N/Z [17]) to allow an important amplification of the initial density fluctuations. And finally, we experimentally detect fragments after secondary decay, which introduces a broadening of the fragment size distribution. Taking into account the accumulation of all these effects on the final extra production of equal-sized fragments, it is clear that any signature that spinodal fragmentation is responsible for the phase transition dynamics can only be what we have called a fossil signature. A full simulation of the spinodal decomposition of quasifused sources using Brownian One-Body dynamics calculations [18] already testified to this fact, with less than $1 \%$ of events with equal-sized fragments. It is the reason why the signature is difficult to observe experimentally.

\section{Experimental results}

The experiment was performed at GANIL (Grand Accélérateur National d'Ions Lourds) and two reactions were used ${ }^{124,136} \mathrm{Xe}+{ }^{112,124} \mathrm{~S} n$ at two bombarding energies, 32 and $45 \mathrm{MeV}$ per nucleon, The beam, impinging on thin targets $\left(530 \mu \mathrm{g} \mathrm{cm}^{-2}\right)$, had an intensity of about 3-5 $10^{7}$ ions per second to avoid event pile-up. Experimental data were collected with the $4 \pi$ multidetector INDRA which is described in detail in $[19,20]$. Accurate particle and fragment identifications were achieved and the energy of the detected products was measured with an accuracy of $4 \%$. Further details can be found in [21-23].

The data used in the analysis were obtained with an on-line multiplicity trigger of 4 or more detected reaction products. The number of such recorded events was between 60 and 80 million events for each colliding system. Quasi-complete events are selected by requiring that at least $80 \%$ of the total charge of the system is measured. We then isolate quasifusion events by using a shape event sorting (see [24] for details). Measured cross-sections corresponding to selected events are $\approx 40 \mathrm{mb}$ at $32 \mathrm{MeV}$ per nucleon and $25 \mathrm{mb}$ at $45 \mathrm{MeV}$ per nucleon. Further details can be found in [24]. Total cross-sections for quasifusion events, taking into account detection efficiency and selection biases, are estimated to be $\sim 250 \mathrm{mb}$ at $32 \mathrm{MeV}$ per nucleon, and $\sim 180 \mathrm{mb}$ at $45 \mathrm{MeV}$ per nucleon.
Twenty years ago an intra-event correlation function called higher order charge correlations [25] was proposed to enlighten any extra production of events with specific fragment partitions. The high sensitivity of the method makes it particularly appropriate to look for small numbers of events as those expected to have kept a memory of spinodal fragmentation properties.

All fragments of one event with fragment multiplicity $M=\sum_{Z} n_{Z}$, where $n_{Z}$ is the number of fragments with charge $Z$ in the partition, are taken into account. By means of the normalized first order:

$$
\langle Z\rangle=\frac{1}{M} \sum_{Z} n_{Z} Z
$$

and second order:

$$
\sigma_{Z}^{2}=\frac{1}{M} \sum_{Z} n_{Z}(Z-\langle Z\rangle)^{2}
$$

moments of the fragment charge distribution in the event, one may define the correlation function $(\mathrm{CF})$ :

$$
1+R\left(\sigma_{Z},\langle Z\rangle\right)=\left.\frac{Y\left(\sigma_{Z},\langle Z\rangle\right)}{Y^{\prime}\left(\sigma_{Z},\langle Z\rangle\right)}\right|_{M}
$$

Here, the numerator $Y\left(\sigma_{Z},\langle Z\rangle\right)$ is the yield of events with given $\langle Z\rangle$ and $\sigma_{Z}$ values. Because the measurement of the charge belonging to a given event is not subject to statistical fluctuations, we use here expression (2) rather than the "nonbiased estimator" of the variance, $\frac{1}{M-1} \sum_{Z} n_{Z}(Z-$ $\langle Z\rangle)^{2}$, as proposed in [25]. The denominator $Y^{\prime}\left(\sigma_{Z},\langle Z\rangle\right)$ represents the uncorrelated yield. Its retained evaluation is based on the "intrinsic probability", intr $P_{Z}$, of emission of a given charge. A unique set of intrinsic probabilities exists only if the chosen event sample is extracted from singlesource events. The denominator partition probabilities are built to take into account in a combinatorial way the trivial correlations due to charge conservation. Thus, this denominator replicates all features of the partitions of the numerator, except the correlations due to other reasons than charge conservation. The probability to observe a given partition $\left(\mathbf{n}:\left(n_{1}, \ldots, n_{Z_{\max }}\right)\right)$, at a given total multiplicity, $m=\sum_{Z} n_{Z}$, is obtained by the multinomial formula. If the total charge is fixed $\left(Z_{\text {tot }}=\sum_{Z} Z n_{Z}\right)$, the partition probabilities are given by:

$$
P(\mathbf{n} \mid m)=\alpha m ! \prod_{Z} \frac{{ }^{\mathrm{intr}} P_{Z}^{n_{Z}}}{n_{Z} !} \delta_{Z_{\mathrm{tot}}, \Sigma_{Z} Z n_{Z}},
$$

where $\alpha$ is the normalization constant (so that $\sum_{n} P(\mathbf{n} \mid m)$ $=1$ ) and $\delta$ is the Kronecker symbol. All the details can be found in Refs. $[18,26]$. The intr $P_{Z}$ values are obtained by means of a recursive procedure of minimization which stops when the decreasing between two steps is lower than $10^{-12}$. The minimization criterion is the normalized $\chi^{2}$ between the experimental partition probabilities and the calculated partition probabilities for a considered set of data. The calculated $\chi^{2} / N_{d o f}$ were always in the range 1.0-0.7.

The considered event samples for the study were those with fragment $(\mathrm{Z} \geqq 5$ ) multiplicities, $M$, from 3 to 6 which correspond to higher statistics. Figure 1 of [24] shows one 

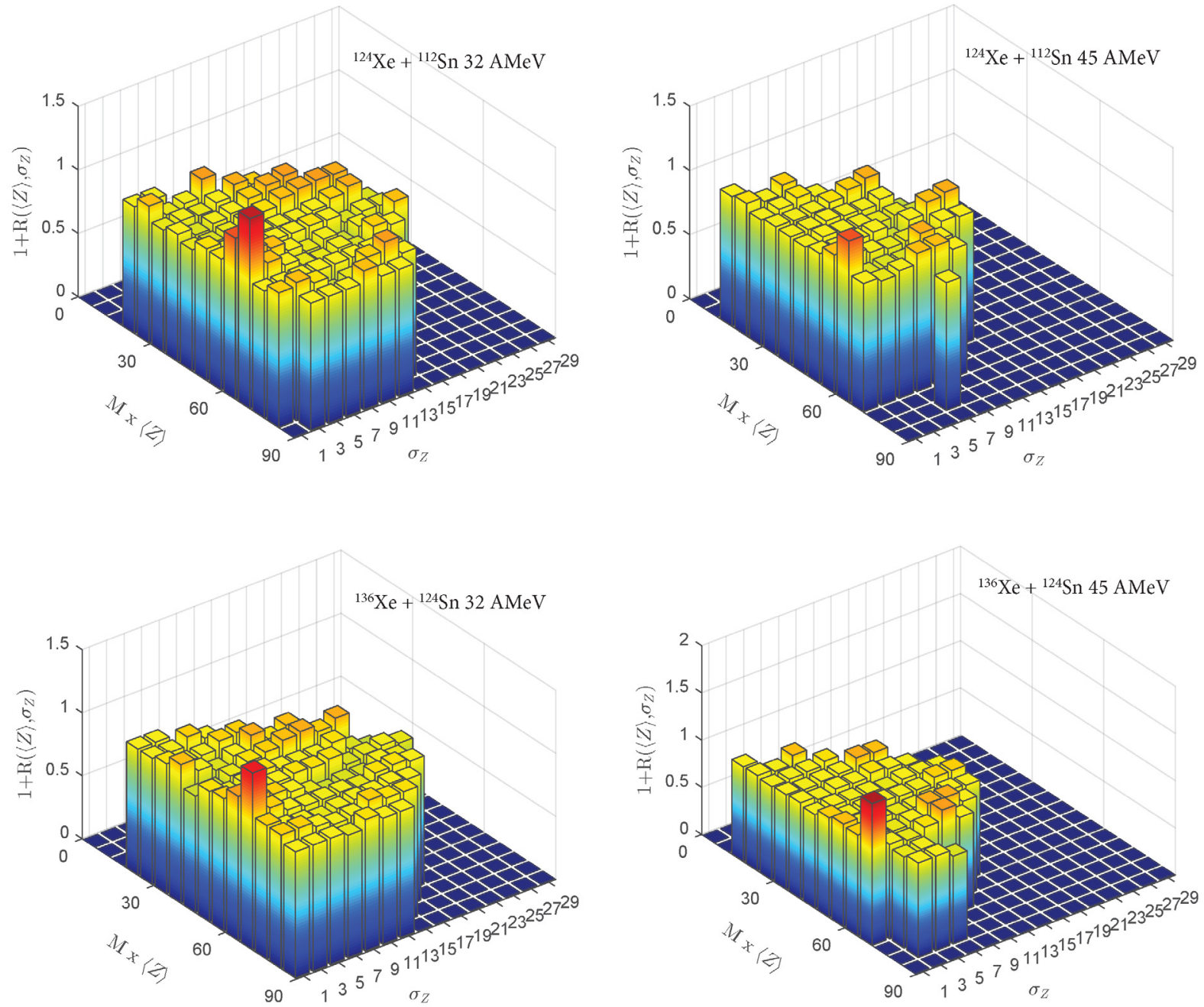

Figure 1. Experimental correlation functions for selected quasifusion events formed in central ${ }^{124,136} \mathrm{Xe}+{ }^{112,124} \mathrm{Sn}$ collisions. Events with fragment multiplicities 3 to 6 are mixed. Correlation functions are calculated for a $\sigma_{Z}$ bin equal to 2 and a $M \times<Z>$ bin equal to 6. The color scale for CFs has a maximum value for 1.6 which corresponds to dark red. From [24].

example on how the experimental fragment charge distributions are faithfully described by using the intrinsic probabilities, ${ }^{\text {intr }} P_{Z}$, which have been calculated independently for each incident energy, for each reaction and for the different fragment multiplicities. $\mathrm{CF}$ values greater than one were observed at very low $\sigma_{Z}(<1)$ but also for $\sigma_{Z}(1-2)$. This observation was used to fix the upper limit at 2 for the $\sigma_{Z}$ of events with nearly equal-sized fragments. The complementary contribution $\left(\sigma_{Z} 1-2\right)$ comes from the broadening of the fragment $Z$ distribution introduced by the deexcitation of primitive fragments (see [24] for details). For the first time, the limited ranges of $\langle Z\rangle$ contributing to $\mathrm{CF}$ peaks were also clearly observed, which verifies what is theoretically expected for finite systems i.e. $M \times\langle Z\rangle \sim$ constant. To better visualize global results, CFs were built for all events of a reaction at a given beam energy, whatever their multiplicity, by summing the correlated yields of all $M$ and by replacing the variable $\langle Z\rangle$ by $M \times\langle Z\rangle$. Uncorrelated yields are then constructed and weighted in proportion to real events of each multiplicity. Fig. 1 summarizes the results. For the four systems CF peaks are observed at low $\sigma_{Z}$. At $32 \mathrm{MeV}$ per nucleon incident energy the neutron poor system exhibits two peaks with confidence levels greater around $6-7 \sigma$ and the neutron rich one peak above $6 \sigma$ and one around $3 \sigma$, which definitively establishes the presence of spinodal fluctuations. At the higher incident energy the two systems have one peak with confidence level above $2 \sigma$. Covered $M \times\langle Z\rangle$ domains are the same $(60-72)$ for both reactions at $32 \mathrm{MeV}$ per nucleon incident energy whereas at higher incident energy the neutron rich system covers a range a little bit higher $(66-72)$ than the neutron poor one $(54-66)$. Finally the percentages of events $\left(Y\left(\sigma_{Z},\langle Z\rangle\right) /\right.$ total number of events) and extra events $\left(\left(Y\left(\sigma_{Z},\langle Z\rangle\right)-Y^{\prime}\left(\sigma_{Z},\langle Z\rangle\right)\right) /\right.$ total number of events) are reported in Table 1 . Within error bars, extra event percentages are similar for both systems at the lower incident energy. At higher incident energy we observe a strong reduction of percentages. The large reduction of the signal observed for the neutron rich system, a factor ten between 32 and $45 \mathrm{MeV}$ per nucleon, can be un- 
Table 1. Percentages of events and extra events with $\sigma_{Z}<2$ for the different incident energies and reactions. Calculated errors are statistical. From [24].

\begin{tabular}{lccc}
\hline $\mathrm{E}(\mathrm{AMeV})$ & reaction & evts & extra evts \\
\hline 32 & ${ }^{124} \mathrm{Xe}+{ }^{112} \mathrm{Sn}$ & 0.27 & $0.068 \pm 0.004$ \\
32 & ${ }^{136} \mathrm{Xe}+{ }^{124} \mathrm{Sn}$ & 0.32 & $0.064 \pm 0.004$ \\
45 & ${ }^{124} \mathrm{Xe}+{ }^{112} \mathrm{Sn}$ & 0.34 & $0.025 \pm 0.003$ \\
45 & ${ }^{136} \mathrm{Xe}+{ }^{124} \mathrm{Sn}$ & 0.030 & $0.0065 \pm 0.0017$ \\
\hline
\end{tabular}

derstood in terms of the expected $N / Z$ influence. Indeed, if spinodal instabilities are at the origin of the dynamics of multifragmentation, as said before, theoretical calculations predict a reduction of instabilities for asymmetric systems in relation with an increase of the instability growth time. More precisely, it is shown in [17] that, for Sn isotopes, the most unstable modes associated to shorter instability growth times $(\simeq 50 \mathrm{fm} / \mathrm{c})$ disappear when $N / Z$ changes from 1.40 to 1.64 . If we consider that quasifusion systems produced by the collisions, with $N / Z$ varying from 1.27 to 1.50 , have to stay long enough in the spinodal region ( $\sim 3$ characteristic times) to allow important amplification of the initial fluctuations, one can qualitatively understand the large extra reduction of the correlation signal for the neutron rich system at high incident energy as coming from insufficient reaction time. Such a situation also favours coalescence of primary fragments. Finally, note that the set of reaction trajectories in the density - temperature plane, close to the border of the spinodal region at $45 \mathrm{MeV}$ per nucleon can be slightly different for the two reactions.

\section{Conclusions}

To summarize on these experimental results one can say that, using charge correlations, the fossil signature of spinodal instabilities i.e. the abnormal presence of nearly equal-sized fragments, even if very low as expected, was definitively established at a confidence level of around 6$7 \sigma$. Associated to this weak signal, it is important to underline again the dominating role of chaotic/stochastic dynamics driven by spinodal instabilities for fragment formation, especially for finite systems. It has to do with beating of modes, breaking of translational symmetry and coalescence during the final step of fragment formation.

From universal fluctuations ( $\Delta$-scaling) the size of the largest fragment of each partition was determined as an order parameter [2, 27-29]. The determination of such an order parameter corresponds to an aggregation scenario which agrees with the process of spinodal fragmentation. Indeed, as far as phase transition dynamics is concerned the aggregation scenario recalls microscopic approaches in which fragments result from spinodal fluctuations oc- curring in the hot, expanding nuclear matter formed in collisions.

\section{References}

[1] P. Chomaz et al. (eds.) Eur. Phys. J. A 30, (2006)

[2] B. Borderie, J.D. Frankland, Prog. Part. Nucl. Phys. 105, 82 (2019)

[3] P. Chomaz et al., Phys. Rev. Lett. 85, 3587 (2000)

[4] M. D’Agostino et al., Phys. Lett. B 473, 219 (2000)

[5] B. Borderie, J. Phys. G: Nucl. Part. Phys. 28, R217 (2002)

[6] N. Le Neindre et al. (INDRA Collaboration), Nucl. Phys. A 795, 47 (2007)

[7] B. Borderie et al. (INDRA Collaboration), Phys. Lett. B 723, 140 (2013)

[8] P. Chomaz et al., Phys. Rev. E 64, 046114 (2001)

[9] E. Bonnet et al. (INDRA Collaboration), Phys. Rev. Lett. 103, 072701 (2009)

[10] M. Colonna, P. Chomaz and A. Guarnera., Nucl. Phys. A 613, 165 (1997)

[11] P. Napolitani and M. Colonna, Phys. Rev. C 96, 054609 (2017)

[12] A. Ono, Phys. Rev. C 59, 853 (1999)

[13] A. Ono et al., Phys. Rev. C 66, 014603 (2002)

[14] E. Bonnet et al., Phys. Rev. C 89, 034608 (2014)

[15] B. Borderie, M.F. Rivet, Prog. Part. Nucl. Phys. 61, 551 (2008) and references therein.

[16] B. Jacquot et al., Phys. Lett. B 383, 247 (1996)

[17] M. Colonna, P. Chomaz and S. Ayik, Phys. Rev. Lett. 88, 122701 (2002)

[18] G. Tăbăcaru et al., Eur. Phys. J. A 18, 103 (2003)

[19] J. Pouthas et al., Nucl. Instr. and Meth. in Phys. Res. A 357, 418 (1995)

[20] J. Pouthas et al., Nucl. Instr. and Meth. in Phys. Res. A 369, 222 (1996)

[21] G. Tăbăcaru et al., INDRA Collaboration, Nucl. Instr. and Meth. in Phys. Res. A 428, 379 (1999)

[22] M. Pârlog et al., INDRA Collaboration, Nucl. Instr. and Meth. in Phys. Res. A 482, 674 (2002)

[23] M. Pârlog et al., INDRA Collaboration, Nucl. Instr. and Meth. in Phys. Res. A 482, 693 (2002)

[24] B. Borderie et al. (INDRA Collaboration), Phys. Lett. B 782, 291 (2018)

[25] L. G. Moretto et al., Phys. Rev. Lett. 77, 2634 (1996)

[26] P. Désesquelles, Phys. Rev. C 65, 034604 (2002)

[27] R. Botet et al., Phys. Rev. E 62, 1825 (2000)

[28] R. Botet et al., Phys. Rev. Lett. 86, 3514 (2001)

[29] J.D. Frankland et al. (INDRA Collaboration), Phys. Rev. C 71, 034607 (2005) 